\section{Mercury and crematorium chimneys}

SIR - The possibility of injury to dentists and their patients from absorption of mercury associated with amalgam fillings has caused considerable concern. But several recent studies ${ }^{1-3}$ have indicated that there is little risk associated with the current widespread use of silver/copper/ tin amalgams ${ }^{4}$, apart from carelessness or with persons of exceptional sensitivity.

Unfortunately, none of the investigators seems to have considered the nature of the final disposal of the filled teeth and their owners. Burial should result in very slow and probably harmless release and dispersion of mercury, but the increasing use of cremation could lead to problems in view of the thermal instability of mercury alloys, the volatility of the free metal, its cumulative toxicity ${ }^{5}$, and the aggregate amounts now involved. Only the possibility of release of radioactive nuclides from implanted pacemakers and therapeutic devices has attracted comment ${ }^{6}$.

I have therefore investigated the thermal decomposition of dental amalgam with samples of well-aged alloy obtained from extracted teeth kindly provided by C. G. W. Wilks. Fragments were powdered, mixed with a little ignited alumina, and then placed, with a thermocouple, in a borosilicate glass test-tube. The tube was then closed with a rubber stopper pierced by a hypodermic needle and a narrow glass tube filled with glass wool coated with palladium chloride. The latter allowed pressures to equalize, but prevented any escape of mercury vapour into the laboratory atmosphere.

The contents of the main tube were then carefully heated with a flame, and equal small volumes of the contained air removed with a microsyringe at measured temperatures in a stepwise progression rising from ambient to $800^{\circ} \mathrm{C}$. The quantity of mercury vapour in each sample was analysed by T. Hackwell and myself by injection into a cold-vapour atomic absorption apparatus specific for this element ${ }^{7}$. A typical result is shown in the figure. It will be seen that decomposition of the dental amalgam was detectable at $200^{\circ} \mathrm{C}$, accelerated above $400^{\circ} \mathrm{C}$, and was essentially complete by $700^{\circ} \mathrm{C}$. Therefore, the contained mercury will be completely released by exposure of teeth containing standard dental fillings (BS 2938; 1985) to the temperatures involved in any form of cremation ${ }^{6}$. It is presumably carried as vapour in the hot gases blown from the chimney, with condensation and/or chemical combination occurring on cooling.

I have been unable to locate any figures for the average weight of mercury now carried by western adults in their fillings, but it is possible to derive an estimate from published statistical surveys ${ }^{8}$. These show that 30 per cent of adults in the United cury and powdered alloy, and mechan-

SIR-On 1 March 1990, a significant portion of the Erebus Glacier tongue calved. This tongue flows from the western side of Mt Erebus into McMurdo Sound, Antarctica, at a point about $20 \mathrm{~km}$ north of New Zealand's Scott Base. Before the calving event, the last $10 \mathrm{~km}$ of the tongue's $16-\mathrm{km}$ length was floating and was, during most of the year, sur-

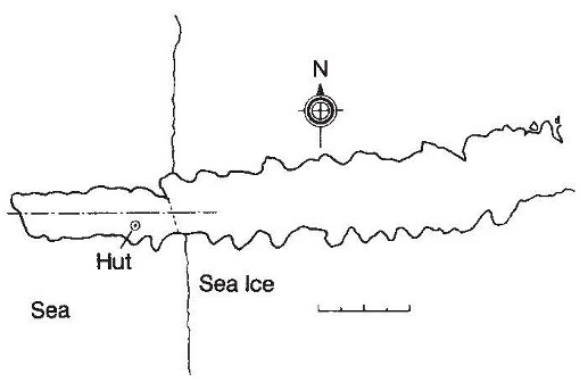

Schematic map of Erebus Glacier tongue showing the site of our hut and the edge of the sea ice on 1 March 1990. The dashed line across the tongue at the sharp bay is the line of fracture of the tongue. Scale bar, $2 \mathrm{~km}$.
Kingdom have lost all their normal complement of 32 teeth, with the rest having on average 7.5 filled - but otherwise satisfactory - teeth. In addition, there is a class for decayed teeth averaging 1.9 per head. Some of these will contain fillings that have failed in their primary purpose, but on the other hand 'fillings' will

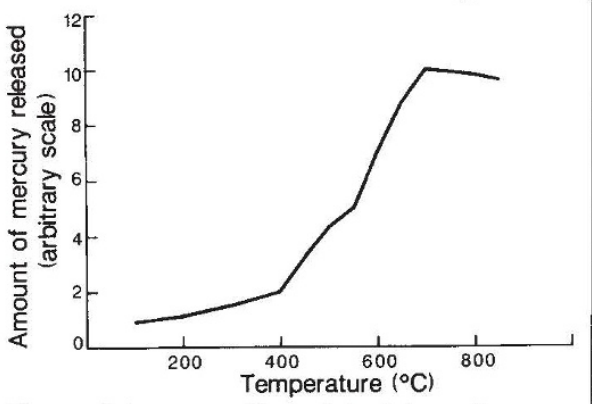

Thermal decomposition of dental amalgam.

also include a proportion of the white mercury-free compositions used for front teeth. It seems reasonable to suggest an average of five amalgam fillings per head for the adult population of the United Kingdom. In 1988, more than 22 million such fillings were emplaced in England and Wales by National Health Service practitioners alone". Even more were placed in previous years.

Nowadays, most dentists purchase commercially prepared sealed plastic ampoules containing encapsulated merically vibrate an ampoule of a suitable size to prepare a batch of amalgam. To obtain some idea of the amount of mercury involved, I opened an unmixed ampoule of medium size and weighed its contents. It contained $0.6 \mathrm{~g}$ of metallic mercury and $0.6 \mathrm{~g}$ of silver-based alloy. Five amalgam fillings of average size would therefore contain 3 g mercury.

Local government records show that one city crematorium carried out an average of 3,723 cremations per annum over the past five years, rising to 3,831 in 1989. Simple multiplication suggests that around $11 \mathrm{~kg}$ of mercury is being released every year from this single (unfiltered) crematorium chimney. As the upper limit for long-term exposure of the general population to mercury vapour in the air has been proposed ${ }^{5}$ as only $1 \mu \mathrm{g} \mathrm{m}^{-3}$, it is imperative that a careful ground and air sampling programme be initiated to assess any possible hazard, and if necessary propose means (activated charcoal filters?) to ameliorate the situation. The safe disposal of extracted filled teeth (or, better, recovery of mercury and silver from them) should also be considered.

AlLAN MiLLS

Department of Geology,

The University,

Leicester LE1 7RH, UK

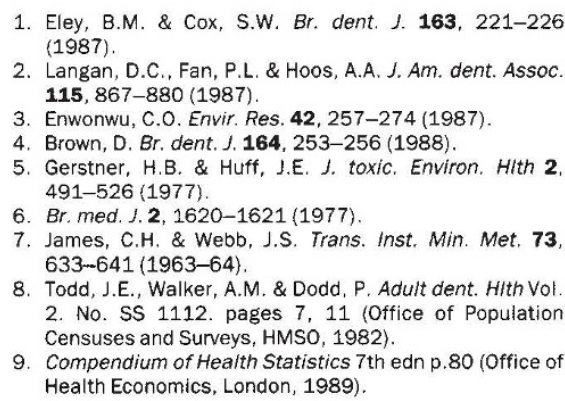

\section{Calving of Erebus Glacier tongue}

rounded by sea ice 1 to $3 \mathrm{~m}$ thick. The tongue's thickness varied from $50 \mathrm{~m}$ at the snout to $300 \mathrm{~m}$ at the point where it is grounded, while its width varied from 0.5 to $2 \mathrm{~km}$ with many large symmetric bays (see figure). The sea depth near the tongue's snout was $300 \mathrm{~m}$. in March 1911 when members of Scott's British Antarctic (Terra Nova) Expedition of 1910-13 noticed that the last $4 \mathrm{~km}$ of the tongue had broken off during a gale. The resulting tabular iceberg drifted around in the McMurdo Sound for several months before becoming grounded on the western side of McMurdo Sound ${ }^{1}$. A similar calving was deduced by Holdsworth to have occurred during the 1940 s (ref. 2).

Recently, we were informed by the 'wintering over' staff at Scott Base that the Erebus Glacier tongue had on 1 March 1990 calved at the sharp bay near the hut containing our equipment used for monitoring the strain of the tongue over the past 5 years (see figure). A few days previously the sea ice surrounding the tongue
The tongue was first observed to calve 